\title{
Desempenho de frangos de corte alimentados com dietas com redução energética contendo diferentes tipos de emulsificantes
}

\section{Performance of broilers fed with energy reduction diets containing different types of emulsifiers}

\author{
Vitor Colossal da Silva ${ }^{1}$, Sanderson Santos da Fonseca ${ }^{2}$, Jean Kaique Valentim ${ }^{3}$, Adriano Geraldo ${ }^{2}$ \\ ${ }^{1}$ Instituto Federal do Norte de Minas Gerais (IFNMG), Montes Claros, MG, Brasil \\ 2 Instituto Federal Minas Gerais (IFMG), Bambuí, MG, Brasil \\ ${ }^{3}$ Universidade Federal dos Vales do Jequitinhonha e Mucuri (UFVJM), Diamantina, MG, Brasil
}

\section{Resumo}

Objetivou-se avaliar a efetividade de emulsificantes comerciais em dietas com redução energética para frangos de corte sobre os parâmetros de desempenho. Foram utilizadas 1152 aves da linhagem comercial COBB 500, distribuídas em 48 parcelas experimentais constituídas por 24 animais (12 machos e 12 fêmeas). Foi utilizado um delineamento em blocos casualizados, com seis tratamentos, sendo dois blocos com quatro repetições cada, totalizando oito repetições por tratamento. A alimentação das aves foi à vontade, tipo farelada, à base de milho e farelo de soja. Foram avaliados o ganho de peso, o consumo de ração, a conversão alimentar e o peso médio no $7^{\circ}, 21^{\circ}$, $35^{\circ}$ e $42^{\circ}$ dia de vida das aves. Os dados coletados foram submetidos à análise de variância, e as médias comparadas pelo teste de Tukey a $5 \%$ de probabilidade, através do programa estatístico SISVAR. O uso do emulsificante B à base de polissorbato melhorou o ganho de peso e o peso médio na fase inicial $(\mathrm{p}<0,05)$. Para a fase de 1 a 42 dias de idade, a redução energética de $60 \mathrm{kcal} / \mathrm{kg}$ pode ter sido insuficiente para a efetividade do produto. Na fase inicial (1 a 21 dias de idade) recomenda-se o uso do emulsificante $\mathrm{B}$, à base de polissobarto, devido aos melhores resultados de desempenho, não havendo efeitos da adição de diferentes emulsificantes sobre o desempenho no período total.

Palavras-chave: Aditivos. Emulsificação de gorduras. Energia da dieta. Redução energética.

\begin{abstract}
The objective of this study was to evaluate the effectiveness of commercial emulsifiers in diets with energy reduction for broilers on the performance parameters. A total of 1152 poultry of the Cobb 500 commercial strain, distributed in 48 experimental plots made up of 24 animals (12 males and 12 females) were used. A randomized complete block design was used, with six treatments being two blocks with four
\end{abstract}


replicates each, totaling eight replications per treatment. Feeding of poultry was free, crumbled type, with corn and soybean meal. The weight gain, feed intake, feed conversion and average weight in 7 th, 21st, 35th and 42nd days of life were evaluated. The data collected weresubmitted toanalysis of variance, and the means were compared by the Tukey test at 5\% probability, using the statistical program SISVAR. The use of polysorbate-based emulsifier B improved weight gain and average weight in the initial phase $(p<0.05)$. For the phase from 1 to 42 days of age the energy reduction of 60 $\mathrm{kcal} / \mathrm{kg}$ may have been insufficient for the effectiveness of the product. In the initial phase (1 to 21 days of age) it is recommended to use emulsifier $B$, based on polissobarto, due to the best performance results, there being no effects of adding different emulsifiers on the performance in the total period.

Keywords: Additives. Fat emulsification. Diet energy. Energy reduction.

\section{Introdução}

O Brasil vem se destacando, ao longo dos anos, como um dos maiores produtores de carne de frango do mundo, tornando-se um dos mais importantes países no mercado internacional. Dentro dessa realidade moderna e competitiva, novas técnicas e produtos se fazem importantes para aumentar a competitividade perante os países concorrentes e a produtividade da atividade.

Devido ao crescimento rápido, as linhagens de frangos de corte apresentam uma elevada demanda energética, o que favorece a utilização dos óleos e gorduras na ração, por serem ingredientes que apresentam alta concentração calórica (Macari et al., 2002). Segundo Pacheco (2014), a energia é um dos componentes de maior custo nas dietas para animais de alto desempenho, devido a sua alta densidade energética, sendo que as gorduras e os óleos são fontes importantes de energia na formulação de dietas para frangos de corte.

Visto isso, minimizar os custos de produção com a alimentação é um dos objetivos da avicultura moderna. Para isso, pode-se adicionar emulsificantes nutricionais, que melhoram a digestibilidade e a absorção da gordura, e, desta forma, conseguem melhorar a eficiência no uso da energia (Rovers, 2013).Emulsificantes são agentes ativos de superfície e compostos anfílicos, de peso molecular médio, que reduzem a tensão superficial e a energia necessária à formação de emulsão(Araújo, 2008). Macromoléculas como proteínas e alguns polissacarídeos podem atuar como emulsificantes, conferindo estabilidade à emulsão por longos períodos.

A adição de emulsificantes às rações pode ser utilizada como estratégia para aumentar a metabolização dos lipídios e, consequentemente, a eficiência energética dos animais, o que resulta em menor custo associado à alimentação e contribui para uma forma mais econômica e sustentável de produção de animais de (Rovers, 2013).

A utilização de um emulsificante alimentar provou ser uma ferramenta para melhorar a digestibilidade da gordura em aves na fase inicial de produção, comparando dietas com emulsificantes exógenos (Roy et al., 2010), pois tornam os glóbulos de gordura mais disponíveis, reforçando a formação de micelas.

Atualmente, no mercado mundial há diversos emulsificantes, no entanto, é importante saber o valor de solubilidade ou hidrossolúbilidade do produto. Quanto mais baixo o equilíbrio, mais lipofílico é o emulsificante (Smulders, 2009). Portanto, devido ao exposto, objetivou-se avaliar a efetividade de emulsificantes comerciais em dietas com redução energética para frangos de corte sobre os parâmetros de desempenho.

\section{Material e métodos}

O experimento foi conduzido no Setor de Avicultura do Instituto Federal de Minas Gerais, Campus Bambuí, de outubro a novembro de 2015, com duração total de 42 dias. 0 projeto de pesquisa foi analisado e aprovado pela Comissão de Ética do Uso de Animais da UNIFENAS, sob o parecer 45A/2015.

Foram utilizadas 1152 aves mistas, da linhagem comercial Cobb 500, distribuídas em 48 parcelas experimentais, sendo cada parcela formada por 24 animais (12 machos e 12 fêmeas). Cada parcela experimental era constituída de um boxe telado com 2,2 $\mathrm{m}^{2}$ de área.

Foi utilizado um delineamento em blocos casualizados, sendo dois blocos com quatro 
repetições cada, testando seis tratamentos, sendo oito repetições por tratamento. Os tratamentos foram:

1. Controle Positivo (CP) - dieta basal com níveis de energia recomendados para a fase $(2950,3080$, 3180 e $3230 \mathrm{kcal} / \mathrm{kg}$, respectivamente, para as dietas pré-inicial, inicial, engorda e final;

2. Controle Negativo (CN) - dieta sem a inclusão de emulsificante e com redução no nível energético $(2910,3040,3120$ e $3170 \mathrm{kcal} / \mathrm{kg}$, respectivamente, para as dietas pré-inicial, inicial, engorda e final);

3. Controle Negativo (CN) - dieta com inclusão do emulsificante $\mathrm{A}$, à base de lecitina de soja $(250 \mathrm{~g} / \mathrm{t})$;

4. Controle Negativo (CN) - dieta com inclusão do emulsificante $\mathrm{B}$, à base de polissorbato $(250 \mathrm{~g} / \mathrm{t})$;

5. Controle Negativo (CN) - dieta com inclusão do emulsificante $\mathrm{C}$, à base de proteína, óleos e gordura vegetal $(350 \mathrm{~g} / \mathrm{t})$;

6. Controle Negativo (CN) - dieta com inclusão da associação dos emulsificantes A + B (250g/t de inclusão, sendo $150 \mathrm{~g}$ do $\mathrm{A}$ e $100 \mathrm{~g}$ do $\mathrm{B}$ ).

0 emulsificante A é composto de lecitina de soja $50 \%$ e sílica $50 \%$ - Nível de garantia: lecitina de soja $500 \mathrm{~g} / \mathrm{kg}$. O emulsificante B é composto de polissobarto $12,5 \%$, ricinoleto de glicerila PEG $12 \%$, ácidos graxos vegetais $5 \%$, monoglicerídeos $2,5 \%$, glicerol $17,5 \%$, sílica $22,2 \%$ e sal $27,8 \%$. 0 emulsificante $\mathrm{C}$ é composto de concentrado de proteína e ácidos graxos vegetais $(58,8 \%)$, contendo $29,5 \%$ de proteína bruta, $5 \%$ de matéria mineral, $4,5 \%$ de umidade e $2,2 \%$ de fibra bruta.

Todas as dietas foram isoprotéicas, isoaminoacídicas, isocálcicas e isofosfóricas. Os níveis de inclusão de lipídeos foram os mesmos para os tratamentos $\mathrm{CN}$ e os suplementados com os diferentes emulsificantes.

A ração das aves foi oferecida à vontade, tipo farelada, à base de milho e farelo de soja. As tabelas $1,2,3$, e 4 apresentam a composição das rações utilizadas na alimentação das aves.

O programa de iluminação foi executado de acordo com as recomendações do manual da linhagem a partir do primeiro dia de idade, e o aquecimento inicial das aves realizado por meio de lâmpadas de infravermelho com potência de $150 \mathrm{~W}$.

As mortalidades foram registradas diariamente, computando a pesagem da ração no dia da morte das aves na parcela para correções no consumo/ ave/dia, conforme metodologia de Sakomura e Rostagno (2007).

As pesagens foram realizadas na chegada dos pintinhos, no $7^{\circ}, 21^{\circ}, 35^{\circ}$ e $42^{\circ}$ dias de idade, sempre na parte da manhã. Realizava-se a pesagem de todos os animais por parcela para posterior cálculo do peso médio das aves.

As variáveis de desempenho analisadas foram: ganho de peso (GP), consumo de ração (CR), conversão alimentar (CA) e peso médio (PM). 0 ganho de peso foi calculado descontando a pesagem inicial dos animais. Para CA, utilizou-se para cálculo a divisão do CR médio/ave pelo GP médio/ave no período avaliado.

\section{Análise estatística}

Os dados coletados foram submetidos à análise de variância através do programa estatístico SISVAR (Ferreira, 2000), aplicando o teste Tukey a 5\% de probabilidade.

\section{Resultados}

Pode-se observar na Tabela 5 os dados de desempenho referentes aos períodos de um a sete dias, onde GP e PM não diferiram significativamente entre os tratamentos ( $p>0,05)$.

Houve diferenças significativas $(\mathrm{p}<0,05)$ dos tratamentos sobre a $\mathrm{CA}$ e $\mathrm{CR}$, onde a dieta $\mathrm{CN}$ suplementada com o emulsificante $\mathrm{A}(\mathrm{CN}+\mathrm{A})$ diferiu negativamente dos demais tratamentos, sendo que $\mathrm{CR}$ apresentou maior valor, mas obteve pior conversão alimentar. $\mathrm{O}$ PM ao sétimo dia não diferiu significativamente entre as dietas $(p>0,05)$.

Com relação ao período de 1 a 21 dias, como mostrado na Tabela 6 , houve diferença significativa entre os tratamentos $(\mathrm{p}<0,05)$ em relação ao $\mathrm{GP}$, sendo que a dieta $\mathrm{CN}$ suplementada com o emulsificante $\mathrm{B}(\mathrm{CN}+\mathrm{B})$ apresentou melhores resultados do que as dietas $\mathrm{CN}, \mathrm{CN}+\mathrm{A}$ e $\mathrm{CN}$ suplementada com emulsificante $A+B(C N+A+B)$, gerando resultados superiores em até $7,45 \%$ quando comparada com $\mathrm{CN}$.

Para o período total do experimento $(1$ a 42 dias) também não houve diferenças significativas ( $p>0,05)$, como visto na Tabela 7 . 
Tabela 1 - Composição centesimal da ração pré-iniciai (1 a 7 dias) para frangos de corte mistos Cobb 500 suplementada ou não com emulsificantes comerciais

\begin{tabular}{|c|c|c|c|c|c|c|}
\hline \multirow[b]{2}{*}{ Ingredientes } & \multicolumn{6}{|c|}{ Tratamentos } \\
\hline & $C P$ & $\mathrm{CN}$ & $\mathrm{CN}+\mathrm{A}$ & $C N+B$ & $\mathrm{CN}+\mathrm{C}$ & $C N+A B$ \\
\hline Milho $(\mathrm{kg})$ & 54,183 & 55,122 & 55,122 & 55,122 & 55,122 & 55,122 \\
\hline Farelo de soja $(\mathrm{kg})$ & 36,375 & 36,210 & 36,210 & 36,210 & 36,210 & 36,210 \\
\hline Óleo de soja (kg) & 3,392 & 2,618 & 2,618 & 2,618 & 2,618 & 2,618 \\
\hline Inerte & 0,050 & 0,050 & 0,025 & 0,025 & 0,015 & 0,025 \\
\hline Emulsificante $A^{1}$ & - & - & 0,025 & - & - & - \\
\hline Emulsificante $\mathrm{B}^{2}$ & - & - & - & 0,025 & - & - \\
\hline Emulsificante $\mathrm{C}^{3}$ & - & - & - & - & 0,035 & - \\
\hline Emulsificantes A (15 g) + B (10 g) & - & - & - & - & - & 0,025 \\
\hline Núcleo Pré-Inicial ${ }^{4}$ & 6,000 & 6,000 & 6,000 & 6,000 & 6,000 & 6,000 \\
\hline TOTAL & 100,000 & 100,000 & 100,000 & 100,000 & 100,000 & 100,000 \\
\hline \multicolumn{7}{|l|}{ Composição calculada da ração } \\
\hline Proteína bruta (\%) & 21,00 & 21,00 & 21,00 & 21,00 & 21,00 & 21,00 \\
\hline EM (kcal/kg) & 2950,0 & 2910,0 & 2910,0 & 2910,0 & 2910,00 & 2910,0 \\
\hline Cálcio (\%) & 1,12 & 1,12 & 1,12 & 1,12 & 1,12 & 1,12 \\
\hline Fósforo disponível (\%) & 0,47 & 0,47 & 0,47 & 0,47 & 0,47 & 0,47 \\
\hline Sódio (\%) & 0,19 & 0,19 & 0,19 & 0,19 & 0,19 & 0,19 \\
\hline Lis digestivel (\%) & 1,05 & 1,05 & 1,05 & 1,05 & 1,05 & 1,05 \\
\hline Met digestível (\%) & 0,42 & 0,42 & 0,42 & 0,42 & 0,42 & 0,42 \\
\hline Met + Cisdigestível (\%) & 0,70 & 0,70 & 0,70 & 0,70 & 0,70 & 0,70 \\
\hline Tre digestível (\%) & 0,73 & 0,73 & 0,73 & 0,73 & 0,73 & 0,73 \\
\hline Trip digestivel (\%) & 0,24 & 0,24 & 0,24 & 0,24 & 0,24 & 0,24 \\
\hline
\end{tabular}

Nota: CP - Dieta controle positivo sem emulsificante.

$\mathrm{CN}$ - Dieta controle negativo sem emulsificante.

$\mathrm{CN}+\mathrm{A}$ - Dieta controle negativo suplementada com 0 emulsificante $\mathrm{A}$ (250/ton).

CN + B - Dieta controle negativo suplementada com o emulsificante B (250/ton).

$C N+C$ - Dieta controle negativo suplementada com o emulsificante $C$ (350/ton).

$C \mathrm{~N}+\mathrm{AB}$ - Dieta controle negativo suplementada com 0 emulsificante $\mathrm{A}+\mathrm{B}(250 /$ ton, sendo $150 \mathrm{~g}$ do $\mathrm{A}$ e $100 \mathrm{~g}$ do $\mathrm{B})$.

${ }^{1}$ Emulsificante A - Composição: lecitina de soja 50\%, sílica 50\% - Nível de garantia: lecitina de soja $500 \mathrm{~g} / \mathrm{kg}$.

${ }^{2}$ Emulsificante B - Composição aproximada: polissobarto 12,5\%, ricinoleto de glicerila PEG 12 \%,ácidos graxos vegetais 5\%, monoglicerídeos 2,5\%, glicerol $17,5 \%$, silica $22,2 \%$, sal $27,8 \%$.

${ }^{3}$ Emulsificante C - Composição química: proteína bruta 29,5\%, matéria mineral 5\%, umidade 4,5\%, fibrabruta 2,2\%, concentrado de proteína e mais óleos e gordura vegetal $58,8 \%$.

${ }^{4}$ Níveis de garantia do núcleo inicial: cálcio (mín) $170 \mathrm{~g}$, cálcio (máx) $210 \mathrm{~g}$, fósforo (mín) $50 \mathrm{~g}$, metionina (mín) $22 \mathrm{~g}$, vitamina A (mín) 120.000 U.I, vitamina D3 (mín) 30.000 U.I, vitamina E (mín) 400 U.I, tiamina (B1) (mín) 35 mg, riboflavina (B2) (mín) 130 mg, piridoxina (B6) (mín) 60 mg, vitamina B12 (mín) 300 mcg, vitamina K3 (mín) $30 \mathrm{mg}$, biotina (mín) 1,6 mg, ácido fólico (mín) 20 mg, niacina (mín) 800 mg, pantotenato de cálcio (mín) 200 mg, colina (mín) 4000 mg, sódio (mín) 26g, manganês (mín) 1600 mg, zinco (mín) 1300 mg, cobre (mín) 160 mg, ferro (mín) 630 mg, iodo (mín) 20 mg, selênio (mín) 6 mg, fitase (mín) 10.000 FTU, avilamicina $200 \mathrm{mg}$ e narasina + nicarbazina $1000 \mathrm{mg}+1000 \mathrm{mg}$. 
Tabela 2 - Composição centesimal da ração inicial (8 a 21 dias) para frangos de corte mistos Cobb 500 suplementada ou não com emulsificantes comerciais

\begin{tabular}{|c|c|c|c|c|c|c|}
\hline \multirow[b]{2}{*}{ Ingredientes } & \multicolumn{6}{|c|}{ Tratamentos } \\
\hline & $C P$ & $\mathrm{CN}$ & $\mathrm{CN}+\mathrm{A}$ & $C N+B$ & $\mathrm{CN}+\mathrm{C}$ & $C N+A B$ \\
\hline Milho $(\mathrm{kg})$ & 57,290 & 58,217 & 58,217 & 58,217 & 58,217 & 58,217 \\
\hline Farelo de soja $(\mathrm{kg})$ & 32,083 & 31,928 & 31,928 & 31,928 & 31,928 & 31,928 \\
\hline Óleo de soja (kg) & 4,777 & 4,005 & 4,005 & 4,005 & 4,005 & 4,005 \\
\hline Inerte & 0,050 & 0,050 & 0,025 & 0,025 & 0,015 & 0,025 \\
\hline Emulsificante $\mathrm{A}^{1}$ & - & - & 0,025 & - & - & - \\
\hline Emulsificante $B^{2}$ & - & - & - & 0,025 & - & - \\
\hline Emulsificante $\mathrm{C}^{3}$ & - & - & - & - & 0,035 & - \\
\hline Emulsificantes A (15 g) + B (10 g) & - & - & - & - & - & 0,025 \\
\hline Núcleo Inicial ${ }^{4}$ & 5,800 & 5,800 & 5,800 & 5,800 & 5,800 & 5,800 \\
\hline TOTAL & 100,000 & 100,000 & 100,000 & 100,000 & 100,000 & 100,000 \\
\hline \multicolumn{7}{|l|}{ Composição calculada da ração } \\
\hline Proteína bruta (\%) & 19,27 & 19,27 & 19,27 & 19,27 & 19,27 & 19,27 \\
\hline EM (kcal/kg) & 3080,02 & 3040,01 & 3040,01 & 3040,01 & 3040,01 & 3040,00 \\
\hline Cálcio (\%) & 1,08 & 1,08 & 1,08 & 1,08 & 1,08 & 1,08 \\
\hline Fósforo disponivel (\%) & 0,45 & 0,45 & 0,45 & 0,45 & 0,45 & 0,45 \\
\hline Sódio (\%) & 0,18 & 0,18 & 0,18 & 0,18 & 0,18 & 0,18 \\
\hline Lis digestível (\%) & 0,95 & 0,94 & 0,94 & 0,94 & 0,94 & 0,94 \\
\hline Met digestível (\%) & 0,40 & 0,39 & 0,39 & 0,39 & 0,39 & 0,39 \\
\hline Met + Cisdigestível (\%) & 0,66 & 0,66 & 0,66 & 0,66 & 0,66 & 0,66 \\
\hline Tre digestível (\%) & 0,67 & 0,67 & 0,67 & 0,67 & 0,67 & 0,67 \\
\hline Trip digestível (\%) & 0,21 & 0,21 & 0,21 & 0,21 & 0,21 & 0,21 \\
\hline
\end{tabular}

Nota: CP - Dieta controle positivo sem emulsificante.

$\mathrm{CN}$ - Dieta controle negativo sem emulsificante.

$\mathrm{CN}+\mathrm{A}$ - Dieta controle negativo suplementada com 0 emulsificante A (250/ton).

CN + B - Dieta controle negativo suplementada com o emulsificante B (250/ton).

$C N+C$ - Dieta controle negativo suplementada com o emulsificante C (350/ton).

$C N+A B$ - Dieta controle negativo suplementada com 0 emulsificante $A+B$ (250/ton, sendo $150 \mathrm{~g}$ do $A$ e $100 \mathrm{~g}$ do $B$ ).

${ }^{1}$ Emulsificante A - Composição: lecitina de soja 50\%, silica 50\% - Nível de garantia: lecitina de soja 500,00 g/kg.

${ }^{2}$ Emulsificante B - Composição aproximada: polissobarto 12,5\%, ricinoleto de glicerila PEG 12\%, ácidos graxos vegetais, 5\% monoglicerídeos, $2,5 \%$ glicerol $17,5 \%$, silica 22,2\%, sal 27,8\%.

${ }^{3}$ Emulsificante C - Composição química: proteína bruta 29,5\%, matéria mineral 5,0\%, umidade 4,5\%, fibrabruta2,2\%, concentrado de proteína e mais óleos e gordura vegetal $58,8 \%$.

${ }^{4}$ Níveis de garantia do núcleo inicial: cálcio (mín) $170 \mathrm{~g}$, cálcio (máx) $210 \mathrm{~g}$, fósforo (mín) $50 \mathrm{~g}$, metionina (mín) $22 \mathrm{~g}$, vitamina A (mín) 120.000 U.I, vitamina D3 (mín) 30.000 U.I, vitamina E (mín) 400 U.I, tiamina (B1) (mín) 35 mg, riboflavina (B2) (mín) 130 mg, piridoxina (B6) (mín) 60 mg, vitamina B12 (mín) 300 mcg, vitamina K3 (mín) 30 mg, biotina (mín) 1,6 mg, ácido fólico (mín) 20 mg, niacina (mín) 800 mg, pantotenato de cálcio (mín) 200 mg, colina (mín) 4000 mg, sódio (mín) 26 g, manganês (mín) 1600 mg, zinco (mín) 1300 mg, cobre (mín) 160 mg, ferro(mín) 630 mg, iodo (mín)20mg, selênio(mín) 6mg,fitase(mín) 10.000FTU, avilamicina $200 \mathrm{mg}$ e narasina + nicarbazina $1000 \mathrm{mg}+1000 \mathrm{mg}$. 
Tabela 3 - Composição centesimal da ração de crescimento (22 a 35 dias) para frangos de corte mistos Cobb 500 suplementada ou não com emulsificantes comerciais

\begin{tabular}{|c|c|c|c|c|c|c|}
\hline \multirow[b]{2}{*}{ Ingredientes } & \multicolumn{6}{|c|}{ Tratamentos } \\
\hline & $C P$ & $\mathrm{CN}$ & $\mathrm{CN}+\mathrm{A}$ & $C N+B$ & $\mathrm{CN}+\mathrm{C}$ & $C N+A B$ \\
\hline Milho $(\mathrm{kg})$ & 58,981 & 60,377 & 60,377 & 60,377 & 60,377 & 60,377 \\
\hline Farelo de soja (kg) & 30,230 & 29,993 & 29,993 & 29,993 & 29,993 & 29,993 \\
\hline Óleo de soja (kg) & 5,739 & 4,580 & 4,580 & 4,580 & 4,580 & 4,580 \\
\hline Inerte & 0,050 & 0,050 & 0,025 & 0,025 & 0,015 & 0,025 \\
\hline Emulsificante $A^{1}$ & - & - & 0,025 & - & - & - \\
\hline Emulsificante $B^{2}$ & - & - & - & 0,025 & - & - \\
\hline Emulsificante $\mathrm{C}^{3}$ & - & - & - & - & 0,035 & - \\
\hline Emulsificantes A (15 g) + B (10 g) & - & - & - & - & - & 0,025 \\
\hline Núcleo de Crescimento ${ }^{4}$ & 5,000 & 5,000 & 5,000 & 5,000 & 5,000 & 5,000 \\
\hline TOTAL & 100,000 & 100,000 & 100,000 & 100,000 & 100,000 & 100,000 \\
\hline \multicolumn{7}{|l|}{ Composição calculada da ração } \\
\hline Proteína bruta (\%) & 18,55 & 18,55 & 18,55 & 18,55 & 18,55 & 18,55 \\
\hline EM (kcal/kg) & 3179,99 & 3119,97 & 3119,97 & 3119,97 & 3119,97 & 3119,97 \\
\hline Cálcio (\%) & 0,99 & 0,99 & 0,99 & 0,99 & 0,99 & 0,99 \\
\hline Fósforo disponível (\%) & 0,35 & 0,35 & 0,35 & 0,35 & 0,35 & 0,35 \\
\hline Sódio (\%) & 0,167 & 0,17 & 0,17 & 0,17 & 0,17 & 0,17 \\
\hline Lis digestivel (\%) & 0,90 & 0,90 & 0,90 & 0,90 & 0,90 & 0,90 \\
\hline Met digestível (\%) & 0,35 & 0,35 & 0,35 & 0,35 & 0,35 & 0,35 \\
\hline Met + Cisdigestível (\%) & 0,61 & 0,61 & 0,61 & 0,61 & 0,61 & 0,61 \\
\hline Tre digestível (\%) & 0,64 & 0,64 & 0,64 & 0,64 & 0,64 & 0,64 \\
\hline Trip digestível (\%) & 0,20 & 0,20 & 0,20 & 0,20 & 0,20 & 0,20 \\
\hline
\end{tabular}

Nota: CP - Dieta controle positivo sem emulsificante.

$\mathrm{CN}$ - Dieta controle negativo sem emulsificante.

$C N+A$ - Dieta controle negativo suplementada com o emulsificante A (250/ton).

$C N+B$ - Dieta controle negativo suplementada com o emulsificante B (250/ton).

$C N+C$ - Dieta controle negativo suplementada com o emulsificante $C$ (350/ton).

$C \mathrm{~N}+\mathrm{AB}$ - Dieta controle negativo suplementada com 0 emulsificante $\mathrm{A}+\mathrm{B}(250 /$ ton, sendo $150 \mathrm{~g}$ do $\mathrm{A} \mathrm{e} 100 \mathrm{~g}$ do $\mathrm{B})$.

${ }^{1}$ Emulsificante A - Composição: lecitina de soja 50\%, sílica 50\% - Nível de garantia: lecitina de soja $500 \mathrm{~g} / \mathrm{kg}$.

${ }^{1}$ Emulsificante A - Composição: lecitina de soja 50\%, sílica 50\% - Nivel de garantia: lecitina de soja $500 \mathrm{~g} / \mathrm{kg}$.

${ }^{2}$ Emulsificante B - Composição aproximada: polissobarto 12,5\%, ricinoleto de glicerila PEG 12 \%,ácidos graxos vegetais $5 \%$, monoglicerídeos 2,5\%, glicerol $17,5 \%$, silica $22,2 \%$, sal $27,8 \%$.

${ }^{3}$ Emulsificante C - Composição química: proteína bruta 29,5\%, matéria mineral 5\%, umidade 4,5\%, fibrabruta 2,2\%, concentrado de proteína e mais óleos e gordura vegetal $58,8 \%$.

${ }^{4}$ Níveis de garantia do núcleo inicial: cálcio (mín) $170 \mathrm{~g}$, cálcio (máx) $210 \mathrm{~g}$, fósforo (mín) $50 \mathrm{~g}$, metionina (mín) $22 \mathrm{~g}$, vitamina A (mín) 120.000 U.I, vitamina D3 (mín) 30.000 U.I, vitamina E (mín) 400 U.I, tiamina (B1) (mín) 35 mg, riboflavina (B2) (mín) 130 mg, piridoxina (B6) (mín) 60 mg, vitamina B12 (mín) 300 mcg, vitamina K3 (mín) 30 mg, biotina (mín) 1,6 mg, ácido fólico (mín) 20 mg, niacina (mín) 800 mg, pantotenato de cálcio (mín) 200 mg, colina (mín) 4000 mg, sódio (mín) 26 g, manganês (mín) 1600 mg, zinco (mín) 1300 mg, cobre (mín) 160 mg, ferro (mín) 630 mg, iodo (mín) 20 mg, selênio (mín) 6 mg, fitase (mín) 10.000 FTU, avilamicina $200 \mathrm{mg}$ e narasina + nicarbazina $1000 \mathrm{mg}+1000 \mathrm{mg}$. 
Tabela 4 - Composição centesimal da ração final (36 a 42 dias) para frangos de corte mistos Cobb 500 suplementada ou não com emulsificantes comerciais

\begin{tabular}{|c|c|c|c|c|c|c|}
\hline \multirow[b]{2}{*}{ Ingredientes } & \multicolumn{6}{|c|}{ Tratamentos } \\
\hline & $C P$ & $\mathrm{CN}$ & $\mathrm{CN}+\mathrm{A}$ & $C N+B$ & $\mathrm{CN}+\mathrm{C}$ & $C N+A B$ \\
\hline Milho (kg) & 63,548 & 64,944 & 64,944 & 64,944 & 64,944 & 64,944 \\
\hline Farelo de soja (kg) & 25,685 & 25,448 & 25,448 & 25,448 & 25,448 & 25,448 \\
\hline Óleo de soja (kg) & 5,717 & 4,558 & 4,558 & 4,558 & 4,558 & 4,558 \\
\hline Inerte & 0,050 & 0,050 & 0,025 & 0,025 & 0,015 & 0,025 \\
\hline Emulsificante $\mathrm{A}^{1}$ & - & - & 0,025 & - & - & - \\
\hline Emulsificante $\mathrm{B}^{2}$ & - & - & - & 0,025 & - & - \\
\hline Emulsificante $\mathrm{C}^{3}$ & - & - & - & - & 0,035 & - \\
\hline Emulsificantes A (15 g) + B (10 g) & & & & & & 0,025 \\
\hline Núcleo Final ${ }^{4}$ & 5,000 & 5,000 & 5,000 & 5,000 & 5,000 & 5,000 \\
\hline TOTAL & 100,000 & 100,000 & 100,000 & 100,000 & 100,000 & 100,000 \\
\hline \multicolumn{7}{|l|}{ Composição calculada da ração } \\
\hline Proteína bruta (\%) & 16,82 & 16,82 & 16,82 & 16,82 & 16,82 & 16,82 \\
\hline EM (kcal/kg) & 3230,00 & 3170,00 & 3170,00 & 3170,00 & 3170,00 & 3170,00 \\
\hline Cálcio (\%) & 0,93 & 0,93 & 0,93 & 0,93 & 0,93 & 0,93 \\
\hline Fósforo disponivel (\%) & 0,24 & 0,24 & 0,24 & 0,24 & 0,24 & 0,24 \\
\hline Sódio (\%) & 0,17 & 0,17 & 0,17 & 0,17 & 0,17 & 0,17 \\
\hline Lis digestível (\%) & 0,79 & 0,79 & 0,79 & 0,79 & 0,79 & 0,79 \\
\hline Met digestível (\%) & 0,31 & 0,31 & 0,31 & 0,31 & 0,31 & 0,31 \\
\hline Met + Cisdigestível (\%) & 0,54 & 0,55 & 0,55 & 0,55 & 0,55 & 0,55 \\
\hline Tre digestível (\%) & 0,58 & 0,58 & 0,58 & 0,58 & 0,58 & 0,58 \\
\hline Trip digestível (\%) & 0,18 & 0,18 & 0,18 & 0,18 & 0,18 & 0,18 \\
\hline
\end{tabular}

Nota: CP - Dieta controle positivo sem emulsificante.

$\mathrm{CN}$ - Dieta controle negativo sem emulsificante.

$\mathrm{CN}+\mathrm{A}$ - Dieta controle negativo suplementada com 0 emulsificante A (250/ton).

CN + B - Dieta controle negativo suplementada com o emulsificante B (250/ton).

$C N+C$ - Dieta controle negativo suplementada com o emulsificante C (350/ton).

$C N+A B$ - Dieta controle negativo suplementada com 0 emulsificante $A+B$ (250/ton, sendo $150 \mathrm{~g}$ do $A$ e $100 \mathrm{~g}$ do $B$ ).

${ }^{1}$ Emulsificante A - Composição: lecitina de soja 50\%, sílica 50\% - Nível de garantia: lecitina de soja $500 \mathrm{~g} / \mathrm{kg}$.

${ }^{2}$ Emulsificante B - Composição aproximada: polissobarto 12,5\%,ricinoleto de glicerila PEG $12 \%$,ácidos graxos vegetais $5 \%$, monoglicerídeos 2,5\%, glicerol $17,5 \%$, silica 22,2\%, sal 27,8\%.

${ }^{3}$ Emulsificante C - Composição química: proteína bruta 29,5\%, matéria mineral 5\%, umidade 4,5\%, fibra bruta 2,2\%, concentrado de proteína e mais óleos e gordura vegetal $58,8 \%$.

${ }^{4}$ Níveis de garantia do núcleo inicial: cálcio (mín) $170 \mathrm{~g}$, cálcio (máx) $220 \mathrm{~g}$, fósforo (mín) $30 \mathrm{~g}$, metionina (mín) 13,5 g,vitamina A (mín) 90.000 U.I, vitamina D3 (mín) 24.000 U.I, vitamina E (mín) 300 U.I, tiamina (B1) (mín) 36 mg, riboflavina (B2) (mín) 90 mg, piridoxina (B6) (mín) 40 mg, vitamina B12 (mín) 200 mcg, vitamina K3 (mín) 20 mg, ácido fólico (mín) 10 mg, niacina (mín) 700 mg, pantotenato de cálcio (mín) 200 mg, colina (mín) 4000 mg, sódio (mín) 28g, manganês (mín) 2000 mg, zinco (mín) 1600 mg, cobre (mín) 200 mg, ferro (mín) 800 mg, iodo (mín) 25 mg, selênio (mín) 6 mg, fitase (mín) 10.000 FTU. 
Tabela 5 - Ganho de peso (GP), conversão alimentar (CA), consumo de ração (CR) e peso médio (PM) de frangos de corte mistos submetidos a dietas com diferentes suplementações de emulsificantes comerciais no período de 1 a 7 dias de idade

\begin{tabular}{lccccccc}
\hline & \multicolumn{7}{c}{ Tratamentos } \\
\hline Variável & $\mathrm{CP}^{1}$ & $\mathrm{CN}^{2}$ & $\mathrm{CN}+\mathrm{A}^{3}$ & $\mathrm{CN}+\mathrm{B}^{4}$ & $\mathrm{CN}+\mathrm{C}^{5}$ & $\mathrm{CN}+\mathrm{AB}^{6}$ & $\mathrm{CV}^{7}(\%)$ \\
$\mathrm{GP}(\mathrm{kg})$ & 0,127 & 0,124 & 0,121 & 0,123 & 0,122 & 0,121 & 5,130 \\
$\mathrm{CA}(\mathrm{kg} / \mathrm{kg})^{* *}$ & $1,283^{\mathrm{a}}$ & $1,322^{\mathrm{a}}$ & $1,557^{\mathrm{b}}$ & $1,366^{\mathrm{a}}$ & $1,400^{\mathrm{a}}$ & $1,305^{\mathrm{a}}$ & 9,450 \\
$\mathrm{CR}(\mathrm{kg})^{* *}$ & $0,162^{\mathrm{a}}$ & $0,163^{\mathrm{a}}$ & $0,189^{\mathrm{b}}$ & $0,168^{\mathrm{a}}$ & $0,170^{\mathrm{a}}$ & $0,158^{\mathrm{a}}$ & 8,500 \\
$\mathrm{PM}(\mathrm{kg})$ & 0,167 & 0,164 & 0,161 & 0,163 & 0,162 & 0,162 & 3,900 \\
\hline
\end{tabular}

Tabela 6 - Ganho de peso (GP), conversão alimentar (CA), consumo de ração (CR) e peso médio (PM) de frangos de corte mistos submetidos a dietas com diferentes suplementações de emulsificantes comerciais no período de 1 a 21 dias de idade

\begin{tabular}{lccccccc}
\hline \multicolumn{7}{c}{ Tratamentos } \\
\hline Variável & $\mathrm{CP}^{1}$ & $\mathrm{CN}^{2}$ & $\mathrm{CN}+\mathrm{A}^{3}$ & $\mathrm{CN}+\mathrm{B}^{4}$ & $\mathrm{CN}+\mathrm{C}^{5}$ & $\mathrm{CN}+\mathrm{AB}^{\mathrm{b}}$ & $\mathrm{CV}^{7}(\%)$ \\
$\mathrm{GP}(\mathrm{kg})$ & $0,7720^{\mathrm{ab}}$ & $0,7510^{\mathrm{b}}$ & $0,7630^{\mathrm{b}}$ & $0,8070^{\mathrm{a}}$ & $0,7820^{\mathrm{ab}}$ & $0,7590^{\mathrm{b}}$ & 3,97 \\
$\mathrm{CA}(\mathrm{kg} / \mathrm{kg})^{* *}$ & 1,5973 & 1,5859 & 1,6726 & 1,5656 & 1,6276 & 1,6056 & 5,96 \\
$\mathrm{CR}(\mathrm{kg})^{* *}$ & 1,2320 & 1,1900 & 1,2760 & 1,2610 & 1,2680 & 1,2170 & 4,72 \\
$\mathrm{PM}(\mathrm{kg})$ & $0,8120^{\mathrm{ab}}$ & $0,7910^{\mathrm{b}}$ & $0,8040^{\mathrm{b}}$ & $0,8470^{\mathrm{a}}$ & $0,8220^{\mathrm{ab}}$ & $0,8000^{\mathrm{b}}$ & 3,77 \\
\hline
\end{tabular}

Tabela 7 - Ganho de peso (GP), conversão alimentar (CA), consumo de ração (CR) e peso médio (PM) de frangos de corte mistos submetidos a dietas com diferentes suplementações de emulsificantes comerciais no período de 1 a 42 dias de idade

\begin{tabular}{lccccccc}
\hline \multicolumn{7}{c}{ Tratamentos } \\
\hline Variável & $\mathrm{CP}^{1}$ & $\mathrm{CN}^{2}$ & $\mathrm{CN}+\mathrm{A}^{3}$ & $\mathrm{CN}+\mathrm{B}^{4}$ & $\mathrm{CN}+\mathrm{C}^{5}$ & $\mathrm{CN}+\mathrm{AB}^{6}$ & $\mathrm{CV}^{7}(\%)$ \\
$\mathrm{GP}(\mathrm{kg})$ & 2,7940 & 2,6980 & 2,8270 & 2,7380 & 2,7990 & 2,7600 & 3,15 \\
$\mathrm{CA}(\mathrm{kg} / \mathrm{kg})^{* *}$ & 1,6475 & 1,7188 & 1,6663 & 1,7050 & 1,6900 & 1,6913 & 3,24 \\
$\mathrm{CR}(\mathrm{kg})^{* *}$ & 4,6000 & 4,6280 & 4,7070 & 4,6650 & 4,7270 & 4,6600 & 2,04 \\
$\mathrm{PM}(\mathrm{kg})$ & 2,8340 & 2,7390 & 2,8660 & 2,7790 & 2,8390 & 2,8000 & 3,11 \\
\hline
\end{tabular}

Nota Tabelas 5, 6 e 7 :

${ }^{* *}$ Médias seguidas de letras diferentes na mesma linha são diferentes estatisticamente pelo teste Tukey $(p<0,05)$.

${ }^{1} \mathrm{CP}$ - dieta controle positivo sem emulsificante.

${ }^{2} \mathrm{CN}$ - dieta controle negativo sem emulsificante.

${ }^{3} \mathrm{CN}+\mathrm{A}$ - dieta controle negativo suplementada com o emulsificante $\mathrm{A}$ (250/ton).

${ }^{4} \mathrm{CN}+\mathrm{B}$ - dieta controle negativo suplementada com o emulsificante $B(250 /$ ton).

${ }^{5} \mathrm{CN}+\mathrm{C}$ - dieta controle negativo suplementada com o emulsificante $\mathrm{C}(350 /$ ton).

${ }^{6} \mathrm{CN}+\mathrm{AB}$ - dieta controle negativo suplementada com o emulsificante $A+B(250 /$ ton, sendo $150 \mathrm{~g}$ do $\mathrm{A} \mathrm{e} 100 \mathrm{~g}$ do $B$ ).

${ }^{7} \mathrm{CV}$ - coeficiente de variação. 


\section{Discussão}

O maior consumo e pior conversão alimentar dos frangos alimentados com dietas suplementadas com o emulsificante A foi observado somente no período de 1 a 7 dias de idade, não ocorrendo nas demais fases analisadas. Este resultado contraria os encontrados por Papadopoulos et al. (2014), que afirmam que dietas convencionais com adição de emulsificantes melhoram a conversão alimentar. Cho et al. (2012), trabalhando com redução energética em dietas para frangos de corte, também observaram que a inclusão de emulsificantes exógenos melhorou o ganho de peso médio dos animais.

Segundo Tancharoenrat et al. (2014), os frangos de corte apresentam alguns limites para a digestão e absorção de lipídeos, sendo que estes induzem as aves na maturidade de seu aparato enzimático, que na fase inicial ainda está em desenvolvimento. Assim, a baixa produção de enzimas para a digestão de lipídios pode prejudicar a digestão dos demais componentes, como proteína e carboidrato, dificultando a absorção dessa fração da dieta e, como consequência, prejudicando o desempenho. Esta limitação provoca uma incapacidade de formar micelas mistas no lúmen intestinal, o que diminui ainda mais a digestão das gorduras e absorção de nutrientes nesta fase de produção (Zhang et al., 2011).

As variáveis CA e CR não apresentaram diferença significativa em nenhum tratamento ( $\mathrm{p}>0,05)$, contrariando $\mathrm{Hu}$ et al. (2012), que utilizando dietas em níveis de energia metabolizável para frangos de $1(12,13 \mathrm{MJ} / \mathrm{kg})$ a 21 dias de idade $(11,92 \mathrm{MJ} / \mathrm{kg})$ e para frangos de $22(12,96 \mathrm{MJ} / \mathrm{kg})$ a 49 dias $(12,65$ $\mathrm{MJ} / \mathrm{kg}$ ), obteviveram melhora na eficiência alimentar.

0 peso médio ao $21^{\circ}$ dia foi melhor significativamente $(p<0,05)$ na dieta $C N+B$, comparada com as dietas $\mathrm{CN}, \mathrm{CN}+\mathrm{A}$ e $\mathrm{CN}+\mathrm{A}+\mathrm{B}$, gerando resultados superiores em até $7,07 \%$ quando comparada com $\mathrm{CN}$. As demais dietas $(\mathrm{CP}$ e $\mathrm{CN}+\mathrm{C})$ não diferiram significativamente.

Através dos resultados, pode-se dizer que a dieta $\mathrm{CN}+\mathrm{B}$ possibilitou melhores resultados nesses níveis de concentração do que as demais, apontando uma possível superioridade da ação do emulsificante polissorbato sobre a lecitina de soja e os emulsificantes proteicos. 0 modo de ação dos emulsificantes consiste em aumentar a superfície ativa das gorduras, permitindo a ação da lipase, que hidrolisa as moléculas de triglicerídeos em ácidos graxos e monoglicerídeos, favorecendo a formação de micelas compostas de produtos da lipólise (Guerreiro Neto et al. 2011).

Yalcinlaya (2005) observou que 1\% de lecitina adicionada a óleo de girassol melhorou o GP de frangos aos 42 dias de idade, o que não aconteceu na presente pesquisa. Araújo et al. (2004) observaram que o óleo de frango proporcionou melhor ganho de peso e tendência de melhor conversão alimentar no período de 1 a 21 dias de idade, sendo que estes resultados não diferiram do óleo de linhaça e foram superiores aos óleos de soja e canola.

No período de 1 a 21 dias, devido ao baixo aparato enzimático atuante na bile, a utilização de emulsificantes em dietas com redução energética foi eficaz por manter o desempenho semelhante a dietas sem redução de energia. No período de 1 a 42 dias, contudo, essa melhora não foi verificada, pois este aparato é desenvolvido na ave com o passar do tempo.

No período de 1 a 42 dias de idade, as dietas utilizadas não influenciaram significativamente o desempenho das aves ( $p>0,05)$. Tal fato pode ser explicado pelo fato de a digestibilidade da gordura melhorar com o avançar da idade, à medida que o aparato enzimático se apresenta mais maduro, independentemente de nível e fonte de gordura (Sellet al., 1986).

Outra explicação plausível para este resultado seria que a redução energética nas dietas fornecidas não foi suficiente para a ação do emulsificante, não acarretando prejuízos no desempenho produtivo. 0 efeito observado da adição do emulsificante B no período inicial não se manteve até o final da criação, $\mathrm{e}$ isto se deve possivelmente pela capacidade de ganho compensatório das aves de corte após esta fase de criação. Zampiga et al. (2016) relatam que mesmo com a melhora na conversão alimentar de frangos de corte alimentados com emulsificante, os efeitos sobre os outros parâmetrosprodutivossãolimitados.

Os efeitos do uso de emulsificantes são diversos em toda literatura. Azman e Cftci (2004) observaram que a substituição de óleo degomado de soja por lecitina na dieta de aves não favoreceu o aumento da assimilação da gordura no trato gastrintestinal de animais jovens. Contradizendo este estudo, Gaiotto 
et al. (2000) observaram melhora nos parâmetros de desempenho com a mistura das fontes óleo de soja e sebo bovino em partes iguais, aos 42 dias de idade.

\section{Conclusão}

$\mathrm{Na}$ fase inicial ( 1 a 21 dias de idade) recomendase o uso do emulsificante $\mathrm{B}$, à base de polissobarto, devido aos melhores resultados de ganho de peso e peso médio, não havendo efeitos da adição de diferentes emulsificantes sobre o desempenho produtivo no período total de criação das aves.

\section{Referencências}

AzmanMA, Ciftci M. Effects of replacing dietary fat with lecithin on broiler chicken zootechnical performance. Revue Med Vet. 2004;155(8-9):445-8.

Araujo JMA. Química de Alimentos - Teoria e Prática. 4 ed. Viçosa: Editora UFV; 2008. 596 p.

Araujo LF, Gomes GA, Almeida EG, Araujo CSS, Junqueira OM, Faria DE. Utilização do ácido linoléico conjugado e de diferentes fontes de óleo na dieta de frangos de corte na fase inicial. Rev Bras Cienc Avic. 2004;6(supl):38.

Cho JH, Zhao P, Kim IH. Effects of emulsifier and multienzyme in different energy density diet on growth performance, blood profiles, and relative organ weight in broiler chickens. J Agric Sci (Toronto). 2012;4(10):161-8.

Ferreira DF. Manual do sistema Sisvar para análises estatísticas. Lavras: Universidade Federal de Lavras; 2000. 63 p.

Gaiotto JB, Menten JFM, Racanicci AMC, Iafigliola MC. Óleo de soja, óleo ácido de soja e sebo bovino como fontes de gordura em rações de frangos de corte. Rev Bras Cienc Avic. 2000;2(3):219-27.

Guerreiro Neto AC, Pezzato AC, Sartori JR, Mori C, Cruz VC, Fascina VB, et al. Emulsifier in broiler diets containing different fat sources. Rev Bras Cienc Avic. 2011;13(2):119-25.
Hu L, Wu X, Zeng Q, Ding X, Zhe L, Zhang K. Diets Supplemented With Soybean Lecith in Emulsifier Effect Growth Performance, Nutrient 21 Availability and Serum Biochemical índices of Broilers. Chin J Anim Nutr. 2012;24(10):1928-38.

Macari M, Furlan RL, Gonzales E. Fisiologia Aviária Aplicada a frangos de corte. Jaboticabal: FUNEP/UNESP; 2002. 375 p.

Pacheco MCM. Efeitos da Inclusão na dieta de lisolecitina de soja na resposta produtiva de broilers [dissertação]. Porto: Universidade do Porto; 2014.

Papadopoulos GA, Müller K, Schertling D, Benedetto M. Supplementation of lysolecithin in combination with a multi-non-starch polysaccharides enzyme improves the feed efficiency during the post-weaning period in piglets. Acta Agric Scand A Anim Sci. 2014;64(2):130-6.

Rovers M. Save energy, feed costs with dietary emulsifier. Feedstuffs; 2013 [acesso 20 mar 2018]. Disponível em: https://tinyurl.com/ya44aemj.

Roy A, Haldar S, Mondal S, Ghosh TK. Effects supplemental exogenous emulsifier on performance, nutrient metabolism, and serum lipid profile in broiler chickens. Vet Med Int. 2010;2010:262604.

Sakomura NK, Rostagno HS. Métodos de pesquisa em nutrição de monogástricos. Jaboticabal: Funep; 2007. 283 p.

Sell JL, Krogdahl A, Hanyu N. Influence of age on utilization of supplemental fats by young turkeys. Poult Sci. 1986;65(3):546-54.

Smulders D. Nutrition emulsifiers make most of feed energy. 2009 [acesso 13 mar 2018]. Disponível em: https://tinyurl.com/ybcw4qhd.

Tancharoenrat P, Ravindran V, Zaefarian F, Ravindran G. Digestion of fat and fatty acids along the gastrointestinal tract of broiler chickens. Poult Sci. 2014;93(2):371-9.

Yalcinlaya I. (2005). Possibilities of using lecithin added oil mixture to replace sunflower oil as an energy source in broiler rations. Vet Fak Derg. 2005;52(1):63-8. 
Zampiga M, Meluzzi A, Sirri F. Effect of dietary supplementation of lysophospholipids on productive performance, nutrient digestibility and carcass quality traits of broiler chickens. Ital J Anim Sci. 2016;15(3): 521-8.

Zhang B, Haitao L, Zhao D, Guo Y, Barri A. Effect of fat type and lysophosphatidylcholine addition to broiler diets on performance, apparent digestibility of fatty acids, and apparent metabolizable energy content. Anim Feed Sci Technol. 2011;163(2-4):177-84. 